\title{
Two-pole structures in a relativistic Friedrichs-Lee-QPC scheme
}

\author{
Zhi-Yong Zhou ${ }^{1, \mathrm{a}}$, Zhiguang Xiao ${ }^{2, \mathrm{~b}}$ \\ ${ }^{1}$ School of Physics, Southeast University, Nanjing 211189, People's Republic of China \\ 2 NSFC-SFTP Peng Huanwu Center for Fundamental Theory and Interdisciplinary Center for Theoretical Study, University of Science and \\ Technology of China, Hefei 230026, Anhui, People's Republic of China
}

Received: 5 November 2020 / Accepted: 13 June 2021 / Published online: 26 June 2021

(C) The Author(s) 2021

\begin{abstract}
A general appearance of two-pole structures is exhibited in a relativistic Friedrichs-Lee model combined with a relativistic quark pair creation model in a consistent manner. This kind of two-pole structure could be found when a $q \bar{q}$ state couples to the open-flavor continuum state in the $S$ partial wave. We found that many enigmatic states, such as $f_{0}(500) / \sigma, K_{0}^{*}(700) / \kappa, a_{0}(980), f_{0}(980), D_{0}^{*}(2300)$, $D_{s 0}^{*}(2317)$, and $X(3872)$, together with another higher state for each, all result from this kind of two-pole structures. Furthermore, an interesting observation is that this kind of twopole structure will contribute roughly a total of $180^{\circ}$ phase shift for the scattering process in a single channel approximation. This relativistic scheme may provide more insights into the understanding of the properties of non- $q \bar{q}$ state. It is also suggested that such two-pole structure could be a common phenomenon which deserves studying both from theoretical and experimental perspectives.
\end{abstract}

\section{Introduction}

As is well-known, states in general are related to the poles of the scattering matrix. In hadron physics, there are always cases that two poles appear together and dynamically related to each other in some scattering processes. For example, the $N$ (1405) signal in the $\bar{K} N$ and $\pi \Sigma$ system was proposed to be contributed by two poles dynamically generated on the same Riemann sheet [1-4]. There could also be the cases that the two poles are located on different Riemann sheets and represent the same state, one of them being the resonance pole and the other the shadow pole. A typical example is the $N(1440)$ state which comes with both a second-sheet pole and a third-sheet shadow pole[5,6]. However, there is another case where one of the two poles comes from a seed

\footnotetext{
a e-mail: zhouzhy@seu.edu.cn

be-mail: xiaozg@ustc.edu.cn (corresponding author)
}

state and the other so called "companion pole" [7] is dynamically generated from the interaction between the same seed state and some continuum state. These double poles may be separated far from each other and be regarded as different states. An old example is the Unitary Quark Model proposed by Törnqvist [8] to explain the lowest scalar nonet. This mechanism could be the cause of the generation of the mysterious $X(3872)$ and a heavier $X(3940)$, that is, the $X(3872)$ could be regarded as being dynamically generated by the interaction between the $\chi_{c 1}(2 P)$ state and the continuum $D \bar{D}^{*}$ [9-16]. Similar idea is also used in the studies on the $\psi$ (3770) meson [17]. In present paper, we will show that the appearance of this kind of two-pole structures could be a common phenomenon in hadron spectrum ranging from the states with light quarks to those with heavy quarks, and a simple dynamical origin of these two-pole structure is revealed in a relativistic constituent quark picture.

The quark potential models are usually regarded as a criterion to characterize the observed hadron states for its generally successful predictions [18]. However, a famous longstanding puzzle is about the lightest $0^{+}$scalar mesons which lie below $1.0 \mathrm{GeV}$, while the lowest $0^{+} \operatorname{scalar}(u \bar{u} \pm d \bar{d}) / \sqrt{2}$, $u \bar{s}$, and $s \bar{s}$ states predicted by the quark potential model are at about 1.1-1.5 GeV [18]. The light scalar states observed in experiments are categorized in three groups according to their isospins: (1) Five $I=0$ states: $f_{0}(500), f_{0}(980)$, $f_{0}(1370), f_{0}(1500)$, and $f_{0}(1710)$; (2) Two $I=1 / 2$ states: $K_{0}^{*}(700)$ and $K_{0}^{*}(1430)$; (3) Two $I=1$ states: $a_{0}(980)$ and $a_{0}(1450)$ [19]. The attempt to categorize these scalar states into suitable nonets was disturbed by the controversy about the existence of $f_{0}(500)$ and $K_{0}^{*}(700)$, until their poles were determined by model-independent methods [20-26]. Nowadays, $f_{0}(500), K_{0}^{*}(700), a_{0}(980)$, and $f_{0}(980)$ are suggested to form a non $-q \bar{q}$ nonet, while $f_{0}(1370), K_{0}^{*}(1430)$, $a_{0}(1450)$, and $f_{0}(1500)$ (or $f_{0}(1710)$ ) are assumed to form the $q \bar{q}$ nonets [27]. The lower nonet has an "inverse" mass relation, which could be understood in the tetraquark model 
proposed by Jaffe $[28,29]$. Another method to study these states is to restore the resonance information from the scattering amplitudes in the chiral perturbation theory $(\chi \mathrm{PT})$ with some unitarization schemes. Large $N_{c}$ analyses demonstrate that those states below $1.0 \mathrm{GeV}$ really do not behave like the $q \bar{q}$ states [30-32]. Besides the puzzle of the light scalar states, more recently, some hadron states with heavy quarks, such as $D_{0}^{*}(2300), D_{s 0}^{*}(2317)$, and $X(3872)$, are also puzzling states which could hardly be accommodated in the predicted $q \bar{q}$ states in the quark potential model. Usually, by unitarizing the scattering amplitudes in the heavy quark chiral perturbation theory $(\mathrm{H} \chi \mathrm{PT})$, several groups claimed that they could be regarded as the hadronic molecular states [3337]. Some other attempts were also made in pursuing the idea that they might be dynamically generated due to the coupling of fundamental states to the continuum states [713,38-49]. The basic spirit of these models here is that by coupling a seed $q \bar{q}$ state to the two-meson continuum states, another state could be generated which could be identified as some possible non-q $\bar{q}$ states. Along these lines, we will provide an understanding of all the above possible non- $q \bar{q}$ states together using the unified Friedrichs-Lee-QPC scheme.

In order to do so, relativistic effects should be considered in a consistent way to cover both the light and the heavy mesons. Recently, we proposed the relativistic FriedrichsLee-QPC scheme [50] which combines the exactly solvable relativistic Friedrichs-Lee model [51] and the relativistic quark pair creation (RQPC) model [52] to study both the low lying meson spectrum and the heavy meson spectrum consistently. In the present paper, this scheme will be applied to study the hadron resonant states mentioned above, in order to understand their nature in this unified framework. We will show that all of these states could be dynamically generated by coupling a seed state and a continuum state, and together with the state originated from the seed state for each one, they form two-pole structures.

The paper is organized as follows: The relativistic FriedrichsLee-QPC scheme is briefly reviewed in Sect. 2. Section 3 is devoted to the main numerical results and discussions on these hadron states. The last section is the summary.

\section{A brief review of relativistic Friedrichs-Lee-QPC scheme}

The basic idea of the Friedrichs-Lee model is that, when the coupling between a discrete state and a continuum state is considered, the discrete state will dissolve into the continuum and becomes a resonant state $[53,54]$. There are also other models implementing similar idea in various areas in physics, such as in atomic physics [55] and in quantum optics [56]. The Friedrichs-Lee model is also linked to quantum field the- ory (QFT) in [57] and also used in the context of baryons [58] and thermal systems [59].

In fact, in the Friedrichs-Lee model, besides the pole shifted from the original discrete state, other dynamically generated poles could appear in the scattering amplitude [14, $60]$. The characteristics of these dynamically generated states is that when the coupling is being turned off, they will not go back to the bare discrete state, but move to the singularities of the form factor, which may be located at the infinity or in the complex plane, thus justifying a dynamic nature of these states. The form factor, as a complex analytic function after analytic continuation with respect to one complex variable, as long as it is not a constant, must have one or more singularities somewhere on the complex plane, as the Liouville theorem in complex analysis tells us. Thus, this kind of scenario of the dynamically generated states could be a general mechanism.

This Friedrichs-Lee scheme was extended to a totally relativistic scenario by including the relativistic kinematics and introducing the creation and annihilation operators for a single-particle state and for a two-particle continuum state mimicked by a so-called bilocal field [50,51]. The full Hamiltonian of the system is written down as

$$
\begin{aligned}
P_{0}= & \int \mathrm{d}^{3} \mathbf{p} \beta(E) \mathrm{d} E E B^{\dagger}(E, \mathbf{p}) B(E, \mathbf{p}) \\
& +\int \mathrm{d}^{3} \mathbf{p} \omega(\mathbf{p}) a^{\dagger}(\mathbf{p}) a(\mathbf{p})+\int \mathrm{d}^{3} \mathbf{p} \beta(E) \mathrm{d} E \alpha(E) \\
& \times\left(a(\mathbf{p})+a^{\dagger}(-\mathbf{p})\right)\left(B^{\dagger}(E, \mathbf{p})+B(E,-\mathbf{p})\right),
\end{aligned}
$$

where $\omega(\mathbf{p})$ denotes the energy of the single particle with momentum $\mathbf{p}, a^{\dagger}(\mathbf{p})$ and $a(\mathbf{p})$ being the operators to create or annihilate this single particle. $B^{\dagger}(E, \mathbf{p})$ and $B(E, \mathbf{p})$ are the so-called bilocal field creation operators and annihilation operators [51] introduced to mimic a two-particle state creation and annihilation with total energy $E$ and momentum $\mathbf{p}$. The other internal quantum numbers such as orbital angular momentum and spins are suppressed. $\alpha(E)$ is the coupling form factor between the single-particle state and the two-particle state, while $\beta(E) \mathrm{d} E \mathrm{~d}^{3} \mathbf{p}$ indicates the integration measure of the two-particle state.

The eigenvalue problem is resolved by finding the solution of operator $b^{\dagger}(E, \mathbf{p})$ in

$\left[P_{0}, b^{\dagger}(E, \mathbf{p})\right]=p_{0} b^{\dagger}(E, \mathbf{p})$,

and this equation could be exactly solved by using the standard techniques of Bogoliubov transformation [50,51]. With the exact solution, the elastic scattering $S$-matrix of the twoparticle continuum state can be expressed as

$S\left(E, \mathbf{p} ; E^{\prime}, \mathbf{p}^{\prime}\right)=\delta^{(3)}\left(\mathbf{p}-\mathbf{p}^{\prime}\right) \delta\left(E-E^{\prime}\right)\left(1-2 \pi i \frac{\rho(s)}{\eta_{+}(s)}\right)$, 
in which $\eta_{+}(s)$, the inverse of resolvent function, reads

$$
\begin{aligned}
& \eta_{+}(s)=s-\omega_{0}^{2}-\Pi(s) \\
& \Pi(s)=\int_{s_{t h}} \mathrm{~d} s^{\prime} \frac{\rho\left(s^{\prime}\right)}{s-s^{\prime}+i 0}
\end{aligned}
$$

$s_{t h}$ is the threshold value of $s$, the invariant mass squared of the continuum state, and $\omega_{0}$ is the bare mass of the single particle. The spectral function $\rho(s)$ is defined as $\rho(s)=2 \omega_{0} \frac{k(s) E_{1}(s) E_{2}(s)}{\sqrt{s}} \alpha(s)^{2}$, in which $k(s)$ is the magnitude of the relative momentum of the two particles in their c.m. frame, and $E_{1}(s)$ and $E_{2}(s)$ are their respective energies. As a consequence of introducing the annihilation operators, Eq. (4) depends on $s$ instead of $E$, similar to the relativistic dispersion relation, which is different from its counterpart in the non-relativistic case $[14,61]$. This $\eta_{+}(s)$ function has a right hand cut starting from the threshold, and could be analytically continued to the complex $s$-plane with two Riemann sheets and is then denoted as $\eta(s)$. With the imaginary part of the $\eta(s)$ function being just $\pi i \rho(s)$ above the threshold, it is easy to see that the $S$ matrix is automatically unitary. Since $\eta(s)$ is the denominator of the $S$-matrix, the zero points of the function on the Riemann sheets are just related to the virtualstate, bound-state, and resonance-state poles of the scattering amplitude. The left-hand-cut contribution is not included in this model, thus the crossing symmetry is violated. However, since the left-hand cut only provides a smooth background to the amplitude near the physical region, its effect can be absorbed into the coupling constant and would not have too much effect on the pole positions which are our main interests in this paper. In principle, the scheme could be extended to the cases with multiple continua. Here we consider only the elastic scattering cases with only one continuum state for simplicity.

The coupling form factor $\alpha(s)$ describes how the bare discrete one-particle state interacts with the bare two-particle continuum state. In our previous works $[14,15]$, in the norelativistic Friedrichs model, the nonrelativistic QPC model was used to describe such interactions of the mesons with heavy quarks. The QPC model is related to the QCD since it just parameterizes a kind of quark pair creation process from the vacuum which can be viewed as a sub-process of QCD, which may be important in the decay processes involving light quarks [62-64]. This model is widely used in describing the interaction of mesons in the literature and is tested to be reasonable (see for example [65-67] and the papers citing them). In present work, the RQPC model is adopted to include some relativistic corrections to the QPC model $[50,52]$. Both at the meson level and at the quark level, the relativistic canonical one-particle and two-particle states and their transformation properties under the Lorentz transformation between the c.m. frame and the other frames are taken into account $[52,68,69]$, so that the whole scheme is formulated in a consistent relativistic manner.

In the RQPC model, to describe the bare meson coupling $A \rightarrow B C$, the $S$-matrix is defined as

$S_{f i}=I-2 \pi i \delta\left(E_{f}-E_{i}\right) T$,

and then

$\langle B C|T| A\rangle=\delta^{(3)}\left(\mathbf{P}_{A}-\mathbf{P}_{B}-\mathbf{P}_{C}\right) \mathscr{M}^{m_{j_{A}} m_{j_{B}} m_{j_{C}},}$

where $\left|j_{A}, m_{j_{A}}\right\rangle$ are the quantum numbers of the total angular

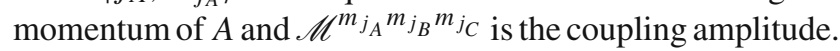
Here, $|A\rangle$ corresponds to the bare discrete state and $|B C\rangle$ corresponds to the bare continuum state in the FriedrichsLee model.

We could write down a relativistic mock state of a meson, with three-momentum $\mathbf{p}$, mass eigenvalue $\tilde{W}$, orbital angular momentum $l$ of two quarks, total spin $s$ of quarks, total angular momentum $j$ and its third component $m_{j}$, as

$$
\begin{aligned}
& \left|\left(\tilde{W},{ }^{2 s+1} l_{j m_{j}}\right)(\mathbf{p})\right\rangle \\
& =\sum_{m_{l} m_{s}} \sum_{m_{1} m_{2}} \sum_{m_{1}^{\prime} m_{2}^{\prime}} \int \mathrm{d}^{3} \mathbf{k} \psi_{l m_{l}}(\mathbf{k}) \\
& \quad \times \phi^{12} \omega^{12}\left|\mathbf{p}_{1}, s_{1} m_{1}^{\prime}\right\rangle \otimes\left|\mathbf{p}_{2}, s_{2} m_{2}^{\prime}\right\rangle \mathscr{D}_{m_{1}^{\prime} m_{1}}^{s_{1}}\left[r_{c}\left(l_{c}(p), k_{1}\right)\right] \\
& \quad \times \mathscr{D}_{m_{2}^{\prime} m_{2}}^{s_{2}}\left[r_{c}\left(l_{c}(p), k_{2}\right)\right]\left\langle s_{1} s_{2} m_{1} m_{2} \mid s m_{s}\right\rangle\left\langle l s m_{l} m_{s} \mid j m_{j}\right\rangle \\
& \quad \times N\left(\mathbf{p}_{1}, \mathbf{p}_{2}, \mathbf{p}, \mathbf{k}\right),
\end{aligned}
$$

where $\mathbf{p}_{1}$ and $\mathbf{p}_{2}$ denote the three momenta of the quark and the antiquark in a general frame, $k_{1}$ and $k_{2}$ their four momenta in the c.m. frame of the meson, $\phi^{12}$ the flavor wave function, $\omega^{12}$ the color wave function of the meson, and $\psi_{l m_{l}}(\mathbf{k})$ is the relative wave function of the quarks in the c.m. frame. $\mathscr{D}_{m_{1}^{\prime} m_{1}}^{s_{1}}[R]$ is the $S U(2)$ matrix representation of rotation group $R$, and $r_{c}\left(l_{c}(p), k_{1}\right)$ represents the Wigner rotation caused by two successive non-collinear Lorentz boosts $l_{c}\left(k_{1}\right)$ and $l_{c}(p)$, which is the key difference between the relativistic meson mock state and the non-relativistic one [67]. $N\left(\mathbf{p}_{1}, \mathbf{p}_{2}, \mathbf{p}, \mathbf{k}\right)$ is the normalization factor.

The interaction operator for the quark pair creation could be expressed as $[52,65]$

$$
\begin{aligned}
T= & -\sqrt{8 \pi} \gamma \int \frac{\mathrm{d}^{3} \mathbf{p}_{3} \mathrm{~d}^{3} \mathbf{p}_{4}}{\sqrt{\varepsilon_{3}\left(\mathbf{p}_{3}\right) \varepsilon_{4}\left(\mathbf{p}_{4}\right)}} \delta^{3}\left(\mathbf{p}_{3}+\mathbf{p}_{4}\right) \\
& \times \sum_{m} \sum_{m_{3} m_{4}}\langle 1, m, 1,-m \mid 0,0\rangle\left\langle 1 / 2, m_{3}, 1 / 2, m_{4} \mid 1,-m\right\rangle \\
& \times \mathscr{Y}_{1}^{m}\left(\frac{\mathbf{p}_{3}-\mathbf{p}_{4}}{2}\right) \phi_{0}^{34} \omega_{0}^{34} b_{m_{3}}^{\dagger}\left(\mathbf{p}_{3}\right) d_{m_{4}}^{\dagger}\left(\mathbf{p}_{4}\right)
\end{aligned}
$$

where $\mathbf{p}_{3}$ and $\mathbf{p}_{4}$ denotes the three-momenta of the quark and the anti-quark produced from the vacuum respectively, $\phi_{0}^{34}$ and $\omega_{0}^{34}$ their flavor and color wave functions, and $\gamma$ 
the quark pair production strength. $\mathscr{Y}_{l}^{m}(\mathbf{p})=|\mathbf{p}|^{l} Y_{l}^{m}(\hat{p})$ is the solid harmonics. $b_{m_{3}}^{\dagger}$ and $d_{m_{4}}^{\dagger}$ are the creation operators of the quark and the anti-quark. Here, the spins and angular momentum of quark and antiquark are combined to form a $J^{P C}=0^{++}$quark pair from the vacuum. The $\gamma$ parameter describes the strength of generating the light quark pairs from the vacuum, which was proposed to be universal in the original QPC model. However, many factors could affect its value for different energy scales. First, since the pair production process must be governed by QCD, the strength would be affected by the running of the QCD coupling with the energy scale. Second, the $\gamma$ parameter may also effectively absorb some interactions which are not included in the quark production processes, such as the left-hand-cut effects. Thus, these effects may renormalize the $\gamma$ parameter such that it may not be the same for low energy and high energy processes. We first try a universal $\gamma$ parameter for both the light and in the heavy cases. We will see that for the system with heavier quarks, choosing another $\gamma$ really causes the pole positions closer to the experimental results.

To connect to the large $N_{c}$ results, we note that by the large $N_{c}$ power counting, the three point amplitude of the mesons in (6) should behave as $O\left(1 / \sqrt{N_{c}}\right)$. Since this amplitude is proportional to $\gamma$, we can effectively put this $N_{c}$ dependence into $\gamma$, i.e. $\gamma \propto 1 / \sqrt{N_{c}}$. Then the large $N_{c}$ limit is the same as $\gamma \rightarrow 0$ limit. Similar method is also used in $[32,48]$.

By the standard derivation from Eqs. (6), (7) and (8), one

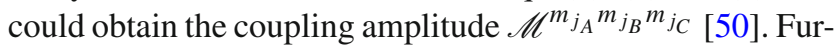
thermore, if we choose the direction of meson $B$ along the $z$-direction, the amplitude with the $B C$ system having relative angular momentum $L$ and the total spin $S$ of meson pair is written down as $[70,71]$

$$
\begin{aligned}
\mathscr{M}^{L S}\left(\mathbf{q}_{z}\right)= & \frac{\sqrt{4 \pi(2 L+1)}}{2 j_{A}+1} \\
& \times \sum_{m_{j_{B}}, m_{j_{C}}}\left\langle L S 0\left(m_{j_{B}}+m_{j_{C}}\right) \mid j_{A}\left(m_{j_{B}}+m_{j_{C}}\right)\right\rangle \\
& \times\left\langle j_{B} j_{C} m_{j_{B}} m_{j_{C}} \mid S\left(m_{j_{B}}+m_{j_{C}}\right)\right\rangle \\
& \times \mathscr{M}^{\left(m_{j_{A}}=m_{j_{B}}+m_{j_{C}}\right) m_{j_{B}} m_{j_{C}}}\left(\mathbf{q}_{z}\right) .
\end{aligned}
$$

Since this amplitude describes the interaction between the discrete state and the continuum, we identify it as the coupling form factor $\alpha_{L S}(s)$ used in Eq. (1) in the relativistic Friedrichs-Lee model in the center of mass system.

The wave functions in the c.m. frame of the quarkantiquark system in Eq. (7) could be obtained by considering the quark-antiquark interaction in the potential model, such as the Godfrey-Isgur (GI) model [18] which is adopted here. The relativistic effect is already implemented in the GI model such that it is consistent with the relativistic Friedrichs-Lee scheme. The wave functions for the bare states are then numerically solved from the GI model. With these wave func- tions, the $\alpha(s)$ can be obtained according to Eq. (9) by calculating the matrix element of $T$ in Eq. (8) as in Eq. (6). The interested reader are referred to our previous work [50] for details. With all these preparations, the emergent physical states could be obtained by finding out the zero points of the $\eta(s)$ function in Eq. (4), which just relate to the poles of the scattering amplitude of the continuum state. Furthermore, the physical phase shifts of the scattering amplitude could also be represented as $\delta(s)=\pi-\operatorname{Arg}[\eta(s)]$. In principle, this relativistic scheme provide a consistent method to study the meson state with light quarks or heavy quarks and could be viewed as including the hadron-loop corrections into the GI's results.

\section{Numerical results and discussions}

The main purpose of this paper is to present the general emergence of two-pole structures in meson spectrum rather than making a systematic fit. Thus, only one of the most important continuum for every bare $q \bar{q}$ state is chosen. Such a simplification to one-continuum scenario could also make the twopole picture clearer by avoiding the complexity introduced in multi-continuum cases [72].

Based on the theoretical background above, the emergence of the two-pole structures in a rather broader spectrum could be studied. The two-pole structure here means that, although there is only one bare seed state interacting with the continuum state, two sets of $S$-matrix poles related with each other appear. One set of poles come from the bare seed state (refers to as "bare" poles) and the other set of poles are dynamically generated by the interaction between the seed and the continuum state (refers to as "dynamical" poles). The "dynamical" poles in general may be originated from the singular point of the interaction form factor, which may be located at infinity or faraway from the seed [60]. Usually, the "bare" poles are a pair of complex conjugate poles representing a resonance. The "dynamical" poles could be a pair of complex conjugate poles, a pair of bound-state and virtual-state poles, or only one bound (or virtual) state, depending on the coupling form factor and its strength. To demonstrate this general phenomenon, only one Okuba-Zweig-Iizuka (OZI) allowed continuum for each bare $q \bar{q}$ state is chosen. Furthermore, the masses of some bare states are slightly deviated from GI's prediction to make the observables, such as the phase shifts, consistent with the measured values in the experiments. This is reasonable, since GI's calculations do not consider the interactions among the mesons. If these interactions are included, and then do the same thing as GI, which is similar to what we are doing here, the masses should be "renormalized" from GI's results. For our purpose, we will consider nine cases, ranging from the lowest scalars to the one with one bottom quarks and the $c \bar{c}\left(2^{3} P_{1}\right)$ cases related to $X(3872)$. 
Table 1 Correspondence of the discrete states and the continuum states as the parameter $\gamma=4.3 \mathrm{GeV}$. The values in the fourth column are the input mass of bare states. Unit is $\mathrm{GeV}$

\begin{tabular}{|c|c|c|c|c|c|c|}
\hline "Discrete" & "Continuum" & GI mass & Input & Poles & Experiment states & PDG values [19] \\
\hline \multirow{2}{*}{$\frac{u \bar{u}+d \bar{d}}{\sqrt{2}}\left(1^{3} P_{0}\right)$} & \multirow[t]{2}{*}{$(\pi \pi)_{I=0}$} & \multirow[t]{2}{*}{1.09} & \multirow[t]{2}{*}{1.3} & $\sqrt{s_{r 1}}=1.34-0.29 i$ & $f_{0}(1370)$ & $1.35^{ \pm 0.15}-0.2^{ \pm 0.05} i$ \\
\hline & & & & $\sqrt{s_{r 2}}=0.39-0.26 i$ & $f_{0}(500)$ & $0.475^{ \pm 0.075}-0.275^{ \pm 0.075} i$ \\
\hline \multirow[t]{2}{*}{$u \bar{s}\left(1^{3} P_{0}\right)$} & \multirow{2}{*}{$(\pi K)_{I=\frac{1}{2}}$} & \multirow[t]{2}{*}{1.23} & \multirow[t]{2}{*}{1.42} & $\sqrt{s_{r 1}}=1.41-0.17 i$ & $K_{0}^{*}(1430)$ & $1.425^{ \pm 0.05}-0.135^{ \pm 0.04} i$ \\
\hline & & & & $\sqrt{s_{r 2}}=0.66-0.34 i$ & $K_{0}^{*}(700)$ & $0.68^{ \pm 0.05}-0.30^{ \pm 0.04} i$ \\
\hline \multirow[t]{2}{*}{$s \bar{s}\left(1^{3} P_{0}\right)$} & \multirow[t]{2}{*}{$K \bar{K}$} & \multirow[t]{2}{*}{1.35} & \multirow[t]{2}{*}{1.68} & $\sqrt{s_{r 1}}=1.71-0.16 i$ & $f_{0}(1710)$ & $1.704^{ \pm 0.012}-0.062^{ \pm 0.009} i$ \\
\hline & & & & $\sqrt{s_{b}}=0.98, \sqrt{s_{v}}=0.19$ & $f_{0}(980)$ & $0.99^{ \pm 0.02}-0.028^{ \pm 0.023} i$ \\
\hline \multirow[t]{2}{*}{$\frac{u \bar{u}-d \bar{d}}{\sqrt{2}}\left(1^{3} P_{0}\right)$} & \multirow[t]{2}{*}{$\pi \eta$} & \multirow[t]{2}{*}{1.09} & \multirow[t]{2}{*}{1.3} & $\sqrt{s_{r 1}}=1.26-0.14 i$ & $a_{0}(1450)$ & $1.474^{ \pm 0.019}-0.133^{ \pm 0.007} i$ \\
\hline & & & & $\sqrt{s_{r 2}}=0.70-0.42 i$ & $a_{0}(980)$ & $0.98^{ \pm 0.02}-0.038^{ \pm 0.012} i$ \\
\hline \multirow[t]{2}{*}{$c \bar{u}\left(1^{3} P_{0}\right)$} & \multirow[t]{2}{*}{$D \pi$} & \multirow[t]{2}{*}{2.4} & \multirow[t]{2}{*}{2.4} & $\sqrt{s_{r 1}}=2.58-0.24 i$ & $D_{0}^{*}(2300)$ & $2.30^{ \pm 0.019}-0.137^{ \pm 0.02} i$ \\
\hline & & & & $\sqrt{s_{r 2}}=2.08-0.10 i$ & & \\
\hline \multirow[t]{2}{*}{$c \bar{s}\left(1^{3} P_{0}\right)$} & \multirow[t]{2}{*}{$D K$} & \multirow[t]{2}{*}{2.48} & \multirow[t]{2}{*}{2.48} & $\sqrt{s_{r 1}}=2.80-0.23 i$ & & \\
\hline & & & & $\sqrt{s_{b}}=2.24, \sqrt{s_{v}}=1.8$ & $D_{s 0}^{*}(2317)$ & $2.317^{ \pm 0.0005}-0.0038^{ \pm 0.0038} i$ \\
\hline \multirow[t]{2}{*}{$b \bar{u}\left(1^{3} P_{0}\right)$} & \multirow[t]{2}{*}{$\bar{B} \pi$} & \multirow[t]{2}{*}{5.76} & \multirow[t]{2}{*}{5.76} & $\sqrt{s_{r 1}}=6.01-0.21 i$ & & \\
\hline & & & & $\sqrt{s_{r 2}}=5.56-0.07 i$ & & \\
\hline \multirow[t]{2}{*}{$b \bar{s}\left(1^{3} P_{0}\right)$} & \multirow[t]{2}{*}{$\bar{B} K$} & \multirow[t]{2}{*}{5.83} & \multirow[t]{2}{*}{5.83} & $\sqrt{s_{r 1}}=6.23-0.17 i$ & & \\
\hline & & & & $\sqrt{s_{b}}=5.66, \sqrt{s_{v}}=5.3$ & & \\
\hline \multirow[t]{2}{*}{$c \bar{c}\left(2^{3} P_{1}\right)$} & \multirow[t]{2}{*}{$D \bar{D}^{*}$} & \multirow[t]{2}{*}{3.95} & \multirow[t]{2}{*}{3.95} & $\sqrt{s_{r 1}}=4.01-0.049 i$ & $X(3940)$ & \\
\hline & & & & $\sqrt{s_{b}}=3.785$ & $X(3872)$ & $3.87169^{ \pm 0.00017}$ \\
\hline
\end{tabular}

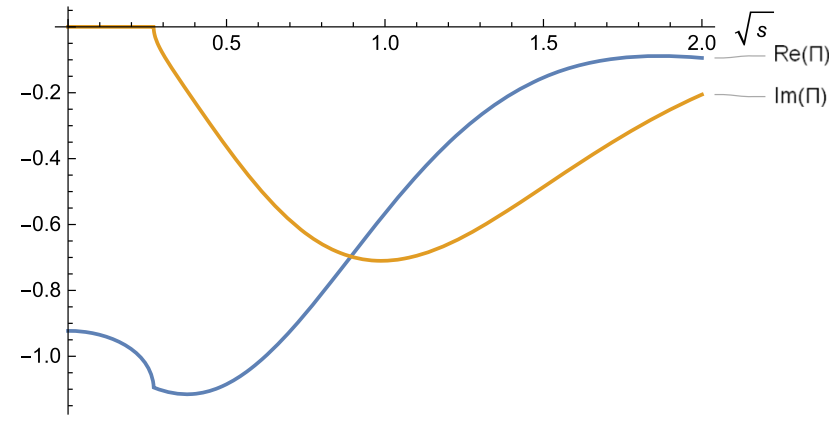

Fig. 1 Real and imaginary parts of $\Pi(s)$ for the scalar $(u \bar{u}+d \bar{d}) / \sqrt{2}$ state

When the parameter $\gamma$ is chosen at about $4.3 \mathrm{GeV}$, the poles of the resolvents are extracted and listed in Table 1. A general consistency between the pole positions, which are defined as $\sqrt{s}=M-i \Gamma / 2$, and the states in the PDG table could be found.

For the scalar $(u \bar{u}+d \bar{d}) / \sqrt{2}$ sector, the real part and imaginary part of self-energy function of $\Pi(s)$ are depicted in Fig. 1. Since there is an exponentially decaying factor in the GI's wave function, the interaction form factor approaches zero fast enough in the high energy limit such that the integration in the self-energy function converges automatically. This is a general feature in our scheme. The resolvent $1 / \eta(s)$ has two pairs of poles on the second Riemann sheet when it

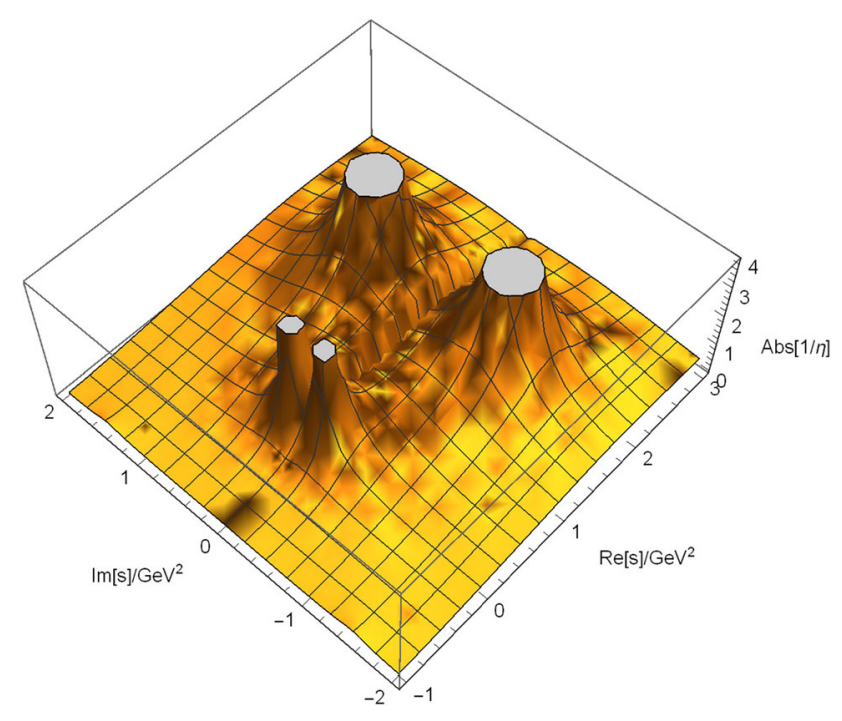

Fig. $2|1 / \eta(s)|$ for the scalar $(u \bar{u}+d \bar{d}) / \sqrt{2}$ on the complex $s$ plane of the second Riemann sheet with $\gamma=4.3 \mathrm{GeV}$

is analytically continued to the complex-s plane, as shown in Fig. 2.

The enigmatic broad $f_{0}(500)$ appears naturally as the "dynamical" state represented by the lighter pair of poles in Fig. 2, while the "bare" one is shifted to become $f_{0}(1370)$ represented by the higher ones in Fig. 2. Similarly, $K_{0}^{*}(700)$ and $K_{0}^{*}(1430)$ are the "dynamical" pole and the "bare" pole 
of scalar $u \bar{s}$ states respectively. The coupling of scalar $s \bar{s}$ bare state and the $K \bar{K}$ continuum, which is OZI-allowed, leads to a "dynamical" bound-state pole just below the $K \bar{K}$ threshold which corresponds to the $f_{0}(980)$ and another virtual-state pole at $0.19 \mathrm{GeV}$ which is too low to be observed. If the $\pi \pi$ continuum coupling to $s \bar{s}$ is considered, which is OZIsuppressed, there will be a new cut from the $\pi \pi$ threshold and the bound state will move onto the second sheet and becomes a narrow resonance pole. This general argument is in agreement with the characteristics of $f_{0}(980)$, which appears as a peak structure in $J / \psi \rightarrow \phi \pi^{+} \pi^{-}$while being nearly invisible in $J / \psi \rightarrow \omega \pi^{+} \pi^{-}$[73]. At the same time, the bare $s \bar{s}$ is shifted onto the complex plane at $1.71-0.16 i \mathrm{GeV}$, whose properties is consistent with the $f_{0}(1710)$, which has a large $s \bar{s}$ components as observed in experiments [74]. To make $f_{0}(980)$ appear in the $\pi \pi$ scattering channel, one must also consider the coupling of the scalar $(u \bar{u}+d \bar{d}) / \sqrt{2}$ to both the $\pi \pi$ and the $K \bar{K}$ continua, and then the $f_{0}(980)$ will come into the $\pi \pi$ interaction through $K \bar{K}$ loop, i.e. through the off-diagonal matrix elements of the inverse resolvent. This will not affect the existence of the $f_{0}(500)$ and $f_{0}(1370)$ poles, but only shift them a little. Since we are confining ourselves to the single channel cases in our present paper, we will leave this for future work.

For the isovector scalar $(u \bar{u}-d \bar{d}) / \sqrt{2}$ state, only coupling to the $\pi \eta$ continuum is considered in this work and a broad resonance at about $0.7 \mathrm{GeV}$ is found. Actually, coupling to $K \bar{K}$ continuum could be comparable with the coupling to $\pi \eta$, and the $K \bar{K}$ threshold could truncate the contribution of a broad resonance pole to produce a narrow peak below the threshold as illustrated in the Flatté effect $[8,44,75]$. A more reasonable description might need a two-channel scenario, which is beyond the scope of this work.

For the scalar $c \bar{u}$ and $c \bar{s}$ states, two-pole structures are also found. Clearly, due to coupling between the scalar $c \bar{s}$ state and the $D K$ continuum, the "dynamical" bound-state pole at $2.24 \mathrm{GeV}$ could correspond to the $D_{s 0}^{*}(2317)$ state. The "bare" pole originated from the $c \bar{s}\left(1^{3} P_{0}\right)$ seed state is located at about $2.80-0.23 i \mathrm{GeV}$. The scalar $c \bar{u}$ state could also produce two poles at $2.08-0.10 i \mathrm{GeV}$ and $2.58-0.24 i$ $\mathrm{GeV}$, in which the lower one is the "dynamical" one and the higher is the "bare" one. Although the experiment only claimed a broad resonance called $D_{0}^{*}(2300)$, it could be contributed by two poles. Further efforts to distinguish these two close poles are quite valuable, because this occasion is different from other two-pole structures whose poles separate from each other. Such a two-pole structure has also been found by the calculations based on unitarizing the $\mathrm{H} \chi \mathrm{PT}$ amplitudes $[35,36,76]$, and they are also comparable with the LQCD simulation [77]. Similarly, their counterparts for the scalar $b \bar{u}$ and $b \bar{s}$ states could also be found here, and the two poles for each case are listed in Table 1. In the unitarized $\mathrm{H} \chi \mathrm{PT}$ approach, the two poles are found to come from
Table 2 The poles' positions for the heavy mesons as $\gamma=3.0 \mathrm{GeV}$. Unit is $\mathrm{GeV}$

\begin{tabular}{lll}
\hline "States" & Bare poles & Dynamical poles \\
\hline$c \bar{u}\left(1^{3} P_{0}\right)$ & $\sqrt{s_{r 1}}=2.39-0.18 i$ & $\sqrt{s_{r 2}}=2.21-0.28 i$ \\
$c \bar{s}\left(1^{3} P_{0}\right)$ & $\sqrt{s_{r 1}}=2.68-0.26 i$ & $\sqrt{s_{b}}=2.32, \sqrt{s_{v}}=1.9$ \\
$b \bar{u}\left(1^{3} P_{0}\right)$ & $\sqrt{s_{r 1}}=5.85-0.26 i$ & $\sqrt{s_{r 2}}=5.62-0.13 i$ \\
$b \bar{s}\left(1^{3} P_{0}\right)$ & $\sqrt{s_{r 1}}=6.11-0.22 i$ & $\sqrt{s_{b}}=5.72, \sqrt{s_{v}}=5.4$ \\
$c \bar{c}\left(2^{3} P_{1}\right)$ & $\sqrt{s_{r}}=3.99-0.05 i$ & $\sqrt{s_{b}}=3.84$ \\
\hline
\end{tabular}

two different $S U$ (3) multiplets in the $S U$ (3) limit. However, after the $S U$ (3) breaking effect is turned on, there should be mixing between these two kinds of $S U$ (3) multiplets and one can no longer distinguish them by different representations. In our approach, we are working with broken $S U(3)$ from the beginning with different $s$ quark and $u / d$ quark masses and the seed should also couple to both triplet and sextet of the continuum. Whether the two-pole structure here is really related to the one in unitarized $\mathrm{H} \chi \mathrm{PT}$ is not trivial and is beyond the scope of our present work.

The $c \bar{c}\left(2^{3} P_{1}\right)$ state could also produce a two-pole structure as we have shown in the non-relativistic Friedrichs model [14, 15]. Here, the "dynamical" pole is also a bound state below the $D \bar{D}^{*}$ threshold but much lower at about $3.78 \mathrm{GeV}$.

The lower results of $X(3872)$ and $D_{s 0}^{*}(2317)$ compared with the observed values might indicate that the $\gamma$ parameter for heavy mesons may be different from the light states as discussed before. If the $\gamma$ parameter is chosen at $3.0 \mathrm{GeV}$ to produce the accurate mass of $D_{s 0}^{*}(2317)$, the masses of the other states are listed in Table 2, which are worth pursuing in future experiments. The results of $c \bar{c}\left(2^{3} P_{1}\right)$ are also improved, closer to our previous works $[14,15]$. Nevertheless, this still demonstrates the existence of the two-pole structure.

Besides the coincidence of the poles' positions with the experiment, further evidences of two-pole structures come from the properties of the scattering phase shifts. A careful analysis of this scheme shows a sum rule for the single channel phase shift here, $\delta(\infty)-\delta\left(s_{t h}\right)=180^{\circ}$ [50]. This means that the two-pole structure contributes a total phase shift of $180^{\circ}$, which should generally not be satisfied if the two poles are independent ${ }^{1}$. This property could be easily verified in $I=1 / 2 \pi K$ scattering phase shift as shown in

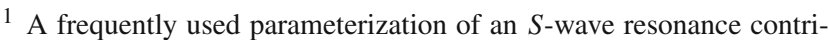
bution to the $S$-matrix is $S=\frac{s-M^{2}-i \rho(s) G_{1}}{s-M^{2}+i \rho(s) G_{1}}$ which contributes $180^{\circ}$ to the phase shift, where $\rho(s)=2 k / \sqrt{s}$ is the kinematic factor, $G_{1}$ being a constant. However, this representation only works for narrow resonances, since besides a pair of resonance poles on the second Riemann sheet, there is also a virtual state pole. A more suitable parametrization is $S=\frac{s-M^{2}-i s \rho(s) G_{2}}{s-M^{2}+i s \rho(s) G_{2}}$ which contributes less than $180^{\circ}$ (see [86] for details).
} 

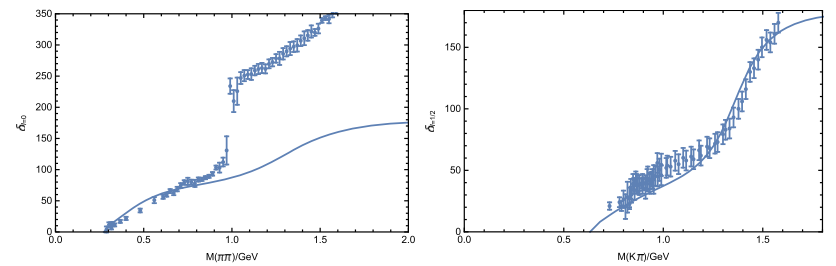

Fig. 3 Comparisons of experimental phase shifts and the theoretical calculations when $\gamma=4.3 \mathrm{GeV}$. The left one is that of $I J=00 \pi \pi$ scattering and the right one is that of $I J=\frac{1}{2} 0 \pi K$ scattering. The solid line is the contribution of two-pole structure

Fig. 3, where the $K_{0}^{*}(700)$ and $K_{0}^{*}(1430)$ contribute a total of roughly $180^{\circ}$ at about $1.7 \mathrm{GeV}$, consistent with the extracted data from the $K \pi$ scattering [78-81]. The phase shift data of the $I J=00 \pi \pi$ scattering also provide some hints to this sum rule, even though it is more complicated for being contributed by both the states generated from $(u \bar{u}+d \bar{d}) / \sqrt{2}$ and $s \bar{s}$ states. As shown in Fig. 3, if the sharp rise of about $180^{\circ}$ at about $1.0 \mathrm{GeV}$ contributed by the narrow $f_{0}(980)$ is removed, the experimental phase shift is just rising smoothly with a total phase shift about $180^{\circ}$ at around $1.6 \mathrm{GeV}$, which may suggest the same sum rule for $f_{0}(500)$ and $f_{0}(1370)$. It is worth noting that the unitarized $\chi \mathrm{PT}$ also suggests that only $\sigma$ or $\kappa$ would not provide a phase shift of $180^{\circ}$ [82].

Some further remarks about the sensitivity of the parameters is in order. Notice that the wave functions of the bare meson states are completely fixed by the GI model whose parameters are totally determined by fitting the meson mass spectrum as in Ref. [18]. Thus, in principle, there is only one free parameter $\gamma$ that denotes the production strength of the $q \bar{q}$ from the vacuum. Once $\gamma$ is chosen, the hadron resonance poles will be determined at the same time. When $\gamma$ changes, all the poles will change simultaneously. As we have mentioned above, to make all the poles' position reasonable we chose $\gamma$ to be at 4.3 or $3.0 \mathrm{GeV}$. The consistency with the experiment values can be seen from Table 1 and 2, which is enough to demonstrate our proposal that the two-pole structure could be a general phenomenon.

It seems that the relative positions of the two poles in different cases are different. However, a numerical experiment could show some common behavior of the pole trajectories as shown in Fig. 4, which shed more light on the general properties of the two-pole structure. For all the $J^{P}=0^{+}$ cases, as the coupling constant $\gamma$ increases from zero, the "bare" pole will move away from the real axis to the second Riemann sheet and becomes a pair of conjugate resonance poles. At the same time, another pair of "dynamical" conjugate poles come from the deep complex $s$-plane, and move towards the real axis and meet on the axis below the threshold becoming a pair of virtual-state poles when the coupling is large enough. As the coupling strength keeps increasing, one of the virtual state pole will move down along the real

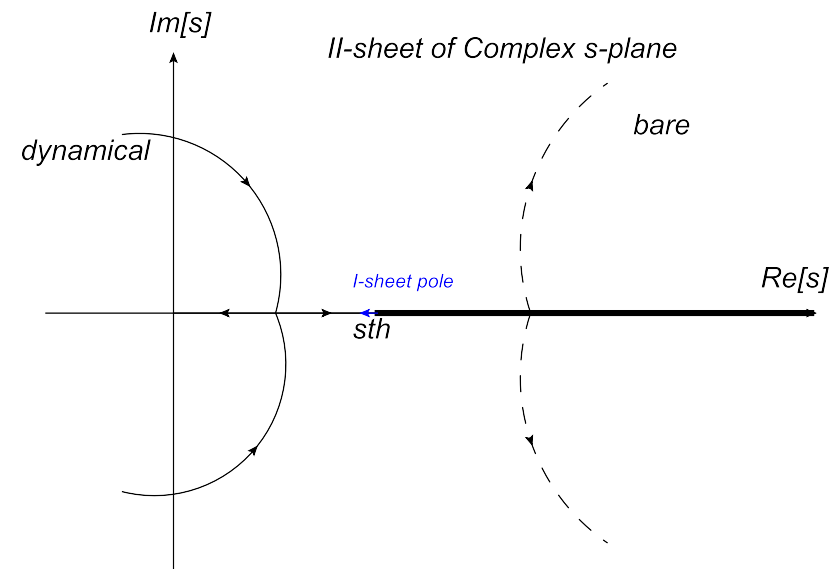

Fig. 4 The general trajectories of two pairs of poles for the two-pole structures on the second Riemann sheet of the $s$-plane as $\gamma$ increases. When $\gamma$ becomes large enough, one of the dynamical pole will move across the threshold and become a bound-state pole as shown in a blue arrow line

axis and the other one moves up across the threshold to the first Riemann sheet and becomes a bound state. On which part of the trajectory the pole position is located will depend on the renormalization effect on $\gamma$, wave functions and the specific parameters such as quark masses in each cases. For the scalar $s \bar{s}, c \bar{s}$, and $b \bar{s}$ states, the coupling is so strong that a bound state and a virtual state are produced for each case, but for other scalar cases the "dynamical" poles remains to be resonances.

Based on the above observation, we would extract some general features in these two-pole phenomena. First, the appearance of a "dynamical" pole needs a nontrivial form factor, which is produced by the coupling of mesons composed of more fundamental quarks. Thus we reach our first general statement: coupling the seed and continuum where all particles involved are composite particles would always produce "dynamical" poles such that this kind of two-pole structure is possible. We have also seen another feature: as the coupling increases from zero, the "dynamical" resonance poles come from far away towards the physical region and may become bound states or nearby virtual states when the coupling becomes stronger. The "dynamical" pole and the "bare" pole may contribute a total phase shift of about $180^{\circ}$ if the single channel approximation can be applied.

Other approaches in the literature also support such a phenomenon. In $\chi \mathrm{PT}$, one can also introduce intermediate $s$-channel resonance in the meson scattering, and after unitarization there could also be dynamically generated accompanying states, such as in [83], where $f_{0}(600) / \sigma, \kappa, a_{0}(980)$ and $f_{0}(980)$ are dynamically generated by coupling a nonet around $1.0 \mathrm{GeV}$ and $1.4 \mathrm{GeV}$ to the low lying pseudoscalars. Another effective field theory method used by Wolkanowski et al. $[16,48,49,84]$ also presented such a phenomenon, 
where the name "companion pole" is used. Their results are similar to ours here. In particular, the pole trajectories and the phase shifts for the $K_{0}^{*}(700)$ and $K_{0}^{*}(1430)$ in Ref. [48] also demonstrate the similar general features listed in the previous paragraph. In quark model, Beveran and Rupp's approach [38-42] is in the same spirit as ours. Instead of using the Friedrichs-Lee model they directly solved the Schrödinger equation with the potential extracted from the QPC model or put by hand, and their relativistic extension of the QPC model $[38,39]$ is different from ours. However, their results and pole trajectories are also similar to ours here and thus demonstrates the general features listed above. More recent works $[47,85]$ using the so called chiral confining Lagrangian also produce similar results for the lightest scalars.

\section{Summary}

To sum up, the interaction between discrete states and the continuum in general may dynamically generate new states and thus results in the two-pole structures, which may be a general mechanism in the strong interactions among hadrons. We show here that the light $0^{+}$scalars $f_{0}(500) / f_{0}(1370)$, $f_{0}(980) / f_{0}(1710), K_{0}^{*}(700) / K_{0}^{*}(1430), a_{0}(980) / a_{0}(1450)$, are this kind of two-pole structures by using the relativistic Friedrichs-Lee-QPC scheme. Furthermore, the two-pole structures $D_{0}^{*}(2210) / D_{0}^{*}(2390), D_{s 0}^{*}(2317) / D_{s 0}^{*}(2680)$ and their counterparts with $b$ quark are also suggested for the future experiments to explore. Though the single channel assumption and the limitation of RQPC may bring in some uncertainty in the pole positions, our results for a wide range of resonances being consistent with the experiment are enough to show the general phenomenon of two-pole structure. Some of the similar two-pole structures were also found in the unitarized $\mathrm{H} \chi \mathrm{PT}$ approach $[35,36,76]$. However, our mechanism here provides a unified constituent quark picture on how these two-pole structures is generated. It is surprising that these two very different approaches converge to the similar results. These are two very different pictures and it is hard to tell which picture is correct or wrong and to make a simple comparison. Or maybe both pictures are partially correct and are just dual to each other in describing the same physics from different facets. Besides the two-pole structures listed above, there could be other two-pole structures generated by the same mechanism which should be paid attention to and be searched for in future experiments. Furthermore, this mechanism could be much more general beyond hadron physics. In molecular physics, atomic physics or condense matter physics, there could also be composite particles scattering where a seed couples with two-particle continuum, and such two-pole structure may also be present. Further exploration in this direction in these areas may reveal new phenomena in future.

Acknowledgements Helpful discussions with Hai-Qing Zhou, ZhiHui Guo, Gang Li, and Feng-kun Guo are appreciated. This work is supported by China National Natural Science Foundation under contract Nos. 11975075, 11575177, and 11947301. Z.Z is also supported by the Natural Science Foundation of Jiangsu Province of China under contract No. BK20171349.

Data Availability Statement This manuscript has no associated data or the data will not be deposited. [Authors' comment: All data generated during this study are contained in this published article.]

Open Access This article is licensed under a Creative Commons Attribution 4.0 International License, which permits use, sharing, adaptation, distribution and reproduction in any medium or format, as long as you give appropriate credit to the original author(s) and the source, provide a link to the Creative Commons licence, and indicate if changes were made. The images or other third party material in this article are included in the article's Creative Commons licence, unless indicated otherwise in a credit line to the material. If material is not included in the article's Creative Commons licence and your intended use is not permitted by statutory regulation or exceeds the permitted use, you will need to obtain permission directly from the copyright holder. To view a copy of this licence, visit http://creativecomm ons.org/licenses/by/4.0/.

Funded by SCOAP ${ }^{3}$.

\section{References}

1. J. Oller, U.G. Meißner, Phys. Lett. B 500, 263 (2001). arXiv:hep-ph/0011146

2. D. Jido, J. Oller, E. Oset, A. Ramos, U. Meißner, Nucl. Phys. A 725, 181 (2003). arXiv:nucl-th/0303062

3. V. Magas, E. Oset, A. Ramos, Phys. Rev. Lett. 95, 052301 (2005). arXiv:hep-ph/0503043

4. T. Hyodo, W. Weise, Phys. Rev. C 77, 035204 (2008). arXiv:0712.1613 [nucl-th]

5. M. Doring, C. Hanhart, F. Huang, S. Krewald, U.-G. Meißner, Nucl. Phys. A 829, 170 (2009). arXiv:0903.4337 [nucl-th]

6. R. Arndt, W. Briscoe, I. Strakovsky, R. Workman, Phys. Rev. C 74, 045205 (2006). arXiv:nucl-th/0605082

7. M. Boglione, M. Pennington, Phys. Rev. D 65, 114010 (2002). arXiv:hep-ph/0203149

8. N.A. Tornqvist, Z. Phys. C 68, 647 (1995). arXiv:hep-ph/9504372

9. Y.S. Kalashnikova, Phys. Rev. D 72, 034010 (2005). arXiv:hep-ph/0506270

10. P.G. Ortega, J. Segovia, D.R. Entem, F. Fernandez, Phys. Rev. D 81, 054023 (2010). arXiv:0907.3997 [hep-ph]

11. M. Takizawa, S. Takeuchi, PTEP 2013, 093D01 (2013). arXiv:1206.4877 [hep-ph]

12. S. Coito, G. Rupp, E. van Beveren, Eur. Phys. J. C 73, 2351 (2013). arXiv:1212.0648 [hep-ph]

13. T. Sekihara, T. Hyodo, D. Jido, PTEP 2015, 063 D04 (2015). arXiv:1411.2308 [hep-ph]

14. Z.-Y. Zhou, Z. Xiao, Phys. Rev. D 96, 054031 (2017). arXiv:1704.04438 [hep-ph] [Erratum: Phys. Rev. D 96, 099905 (2017)]

15. Z.-Y. Zhou, Z. Xiao, Phys. Rev. D 97, 034011 (2018) arXiv:1711.01930 [hep-ph]

16. F. Giacosa, M. Piotrowska, S. Coito, (2019). arXiv:1903.06926 [hep-ph] 
17. S. Coito, F. Giacosa, Nucl. Phys. A 981, 38 (2019). arXiv:1712.00969 [hep-ph]

18. S. Godfrey, N. Isgur, Phys. Rev. D 32, 189 (1985)

19. M. Tanabashi et al. [Particle Data Group], Phys. Rev. D 98, 030001 (2018)

20. I. Caprini, G. Colangelo, H. Leutwyler, Phys. Rev. Lett. 96, 132001 (2006). arXiv:hep-ph/0512364

21. Z. Zhou, G. Qin, P. Zhang, Z. Xiao, H. Zheng et al., JHEP 0502, 043 (2005). arXiv:hep-ph/0406271

22. H.Q. Zheng, Z.Y. Zhou, G.Y. Qin, Z. Xiao, J.J. Wang, N. Wu, Nucl. Phys. A 733, 235 (2004). arXiv:hep-ph/0310293

23. S. Descotes-Genon, B. Moussallam, Eur. Phys. J. C 48, 553 (2006). arXiv:hep-ph/0607133

24. J. Peláez, A. Rodas, Phys. Rev. Lett. 124, 172001 (2020). arXiv:2001.08153 [hep-ph]

25. J.R. Pelaez, Phys. Rept. 658, 1 (2016). arXiv:1510.00653 [hep-ph]

26. D.-L. Yao, L.-Y. Dai, H.-Q. Zheng, Z.-Y. Zhou, (2020). arXiv:2009.13495 [hep-ph]

27. F.E. Close, N.A. Tornqvist, J. Phys. G 28, R249 (2002). arXiv:hep-ph/0204205

28. R.L. Jaffe, Phys. Rev. D 15, 267 (1977)

29. L. Maiani, F. Piccinini, A.D. Polosa, V. Riquer, Phys. Rev. D 71, 014028 (2005). arXiv:hep-ph/0412098

30. J. Oller, E. Oset, J. Pelaez, Phys. Rev. D, 59, 074001 (1999). arXiv:hep-ph/9804209 [Erratum: Phys.Rev.D 60, 099906 (1999), Erratum: Phys.Rev.D 75, 099903 (2007)]

31. Z.-H. Guo, J. Oller, Phys. Rev. D 84, 034005 (2011). arXiv:1104.2849 [hep-ph]

32. J. Pelaez, Phys. Rev. Lett. 92, 102001 (2004). arXiv:hep-ph/0309292

33. F.-K. Guo, P.-N. Shen, H.-C. Chiang, R.-G. Ping, B.-S. Zou, Phys. Lett. B 641, 278 (2006). arXiv:hep-ph/0603072

34. F.-K. Guo, P.-N. Shen, H.-C. Chiang, Phys. Lett. B 647, 133 (2007). arXiv:hep-ph/0610008

35. Z.-H. Guo, U.-G. Meißner, D.-L. Yao, Phys. Rev. D 92, 094008 (2015). arXiv:1507.03123 [hep-ph]

36. M. Albaladejo, P. Fernandez-Soler, F.-K. Guo, J. Nieves, Phys. Lett. B 767, 465 (2017). arXiv:1610.06727 [hep-ph]

37. T.-W. Wu, M.-Z. Liu, L.-S. Geng, E. Hiyama, M.P. Valderrama, Phys. Rev. D 100, 034029 (2019). arXiv:1906.11995 [hep-ph]

38. E. van Beveren, G. Rupp, T. Rijken, C. Dullemond, Phys. Rev. D 27, 1527 (1983a)

39. E. van Beveren, C. Dullemond, T. Rijken, Z. Phys. C 19, 275 (1983b)

40. E. van Beveren, T. Rijken, K. Metzger, C. Dullemond, G. Rupp, J. Ribeiro, Z. Phys. C 30, 615 (1986). arXiv:0710.4067 [hep-ph]

41. E. van Beveren, D. Bugg, F. Kleefeld, G. Rupp, Phys. Lett. B 641 , 265 (2006). arXiv:hep-ph/0606022

42. E. van Beveren, G. Rupp, Phys. Rev. Lett. 91, 012003 (2003). arXiv:hep-ph/0305035

43. K. Heikkila, N.A. Tornqvist, S. Ono, Phys. Rev. D 29, 110 (1984)

44. Z.-Y. Zhou, Z. Xiao, Phys. Rev. D 83, 014010 (2011a). arXiv:1007.2072 [hep-ph]

45. M. Boglione, M.R. Pennington, Phys. Rev. Lett. 79, 1998 (1997). arXiv:hep-ph/9703257

46. P. Geiger, N. Isgur, Phys. Rev. D 47, 5050 (1993)

47. A. Badalian, M. Lukashov, Y.A. Simonov, (2020). arXiv:2001.07113 [hep-ph]

48. T. Wolkanowski, M. Sołtysiak, F. Giacosa, Nucl. Phys. B 909, 418 (2016a). arXiv:1512.01071 [hep-ph]

49. T. Wolkanowski, F. Giacosa, D.H. Rischke, Phys. Rev. D 93, 014002 (2016b). arXiv:1508.00372 [hep-ph]

50. Z.-Y. Zhou, Z. Xiao, Eur. Phys. J. C 80, 1191 (2020). arXiv:2008.02684 [hep-ph]

51. I. Antoniou, M. Gadella, I. Prigogine, G.P. Pronko, J. Math. Phys. 39, 2995 (1998)
52. M.G. Fuda, Phys. Rev. C 86, 055205 (2012)

53. K.O. Friedrichs, Commun. Pure Appl. Math. 1, 361 (1948)

54. T.D. Lee, Phys. Rev. 95, 1329 (1954)

55. P. Facchi, H. Nakazato, S. Pascazio, Phys. Rev. Lett. 86, 2699 (2001)

56. E.T. Jaynes, F.W. Cummings, Proc. IEEE 51, 89 (1963)

57. F. Giacosa, Found. Phys. 42, 1262 (2012). arXiv:1110.5923 [nuclth]

58. Z.-W. Liu, W. Kamleh, D.B. Leinweber, F.M. Stokes, A.W. Thomas, J.-J. Wu, Phys. Rev. Lett. 116, 082004 (2016). arXiv: 1512.00140 [hep-lat]

59. P.M. Lo, F. Giacosa, Eur. Phys. J. C 79, 336 (2019). arXiv:1902.03203 [hep-ph]

60. Z. Xiao, Z.-Y. Zhou, Phys. Rev. D 94, 076006 (2016). arXiv:1608.00468 [hep-ph]

61. Z. Xiao, Z.-Y. Zhou, J. Math. Phys. 58, 072102 (2017). arXiv:1610.07460 [hep-ph]

62. H.G. Dosch, D. Gromes, Phys. Rev. D 33, 1378 (1986)

63. R. Kokoski, N. Isgur, Phys. Rev. D 35, 907 (1987)

64. E.S. Ackleh, T. Barnes, E.S. Swanson, Phys. Rev. D 54, 6811 (1996). arXiv:hep-ph/9604355

65. L. Micu, Nucl. Phys. B 10, 521 (1969)

66. A. Le Yaouanc, L. Oliver, O. Pene, J. Raynal, Phys. Rev. D 8, 2223 (1973)

67. H.G. Blundell, S. Godfrey, Phys. Rev. D 53, 3700 (1996). arXiv:hep-ph/9508264

68. A.J. Macfarlane, J. Math. Phys. 4, 490 (1963). https://doi.org/10. $1063 / 1.1703981$

69. A. McKerrell, Nuovo Cim. 34, 1289 (1964)

70. M. Jacob, G.C. Wick, Ann. Phys. 7, 404 (1959)

71. M. Jacob, G.C. Wick, Ann. Phys. 281, 774 (2000)

72. Z.-Y. Zhou, Z. Xiao, Phys. Rev. D 84, 034023 (2011b). arXiv:1105.6025 [hep-ph]

73. A. Deandrea, R. Gatto, G. Nardulli, A. Polosa, N. Tornqvist, Phys. Lett. B 502, 79 (2001). arXiv:hep-ph/0012120

74. M. Acciarri et al. (L3), Phys. Lett. B 501, 173 (2001). arXiv:hep-ex/0011037

75. S.M. Flatte, Phys. Lett. B 63, 224 (1976)

76. U.-G. Meißner, Symmetry 12, 981 (2020). arXiv:2005.06909 [hep$\mathrm{ph}]$

77. G. Moir, M. Peardon, S.M. Ryan, C.E. Thomas, D.J. Wilson, JHEP 10, 011 (2016). arXiv:1607.07093 [hep-lat]

78. R. Mercer et al., Nucl. Phys. B 32, 381 (1971)

79. P. Estabrooks, R. Carnegie, A.D. Martin, W. Dunwoodie, T. Lasinski, D.W. Leith, Nucl. Phys. B 133, 490 (1978)

80. H. Bingham et al., Nucl. Phys. B 41, 1 (1972)

81. D. Aston et al., Nucl. Phys. B 296, 493 (1988)

82. A. Dobado, J.R. Pelaez, Phys. Rev. D 56, 3057 (1997). arXiv:hep-ph/9604416

83. J.A. Oller, E. Oset, Phys. Rev. D 60, 074023 (1999). arXiv:hep-ph/9809337

84. F. Giacosa, Acta Phys. Polon. Supp. 13, 83 (2020). arXiv:1904.10368 [hep-ph]

85. M.S. Lukashov, Y.A. Simonov, Phys. Rev. D 101, 094028 (2020). arXiv:1909.10384 [hep-ph]

86. H.-Q. Zheng, in International Symposium on Hadron Spectroscopy. Chiral Symmetry and Relativistic Description of Bound Systems (2003), pp. 98-105. arXiv:hep-ph/0304173 